\title{
On the Dynamics of Pressurized and Atmospheric Circulating Fluidized Bed Risers
}

\author{
Michel Y. Louge, Vincent Bricout and Stéphane Martin-Letellier \\ Sibley School of Mechanical Engineering, Cornell University, Ithaca, NY 14853 USA
}

\begin{abstract}
We investigate the effects of gas density and operating conditions on the gas pressure gradient in the upper riser of circulating fluidized beds. By matching five dimensionless parameters, our experiments achieve hydrodynamic similarity with generic high-temperature risers operating at pressures of 1 and $8 \mathrm{~atm}$.

Using momentum balances for the gas and solid phases, we find that, for a wide range of experiments, two parameters capture the dependence of the steady, fully-developed pressure gradients upon the ratio of the mean gas and solid mass flow rates. The first is the ratio of the mean particle slip and superficial gas velocities. Its variations indicate that the "atmospheric" and "pressurized" experiments conform to distinct viscous and inertial regimes.
\end{abstract}

\section{BACKGROUND}

The present paper is a selection of results from a wider study (1). We employ dimensional analysis to simulate the behavior of high-temperature pressurized and atmospheric circulating fluidized bed risers in a laboratory facility operated near ambient pressure and temperature (2). Following Chang and Louge ( $\underline{3})$, we recirculate -rather than discard- fluidization gas mixtures of adjustable density. "Atmospheric" and "pressurized" conditions are reproduced using, respectively, light mixtures of helium and carbon dioxide, and denser mixtures of sulfur hexafluoride and air. Hydrodynamic analogy between the cold bed and a hot industrial reactor is achieved by matching the five dimensionless parameters arising, for example, from the analysis of Anderson and Jackson (4):

$\mathrm{Fr}=\mathrm{u} / \sqrt{\mathrm{g \phi d}}$,

$\mathrm{M}=\mathrm{G} / \rho \mathrm{u}$,

$\operatorname{Ar}=\rho_{\mathrm{S}} \rho\left(\phi \mathrm{d}_{\mathrm{S}}\right)^{3} \mathrm{~g} / \mu^{2}$,

$\mathrm{R}=\rho_{\mathrm{s}} / \rho$,

$\mathrm{L}=\mathrm{D} / \phi \mathrm{d}_{\mathrm{s}}$.

In these expressions, $\rho, \mu$, and $\rho_{\mathrm{s}}$ are the density of the gas, its viscosity, and the material density of the solids, respectively; $u$ is the superficial gas velocity; $G$ is the overall solid flux; $\mathrm{g}$ is the acceleration of gravity; and $\mathrm{D}$ is the riser diameter. Following the suggestion of ( $\underline{3})$, the effective particle diameter is the product of the mean particle Sauter diameter $d_{s}$ and its sphericity $\phi$. Because in this study we employ a single facility, other dimensionless aspect ratios such as the ratio of riser height and diameter are readily matched in all experiments. 
Algebraic manipulations of Eqs. [1] through [5] relate the operating conditions and properties of the laboratory facility to those in the hot industrial reactor that it models. In this study, we simulate a generic coal combustor with $\rho_{\mathrm{s}}=1500 \mathrm{~kg} / \mathrm{m}^{3}, \mu=4.210^{-5} \mathrm{~kg} / \mathrm{m} . \mathrm{s}$ and $\phi \mathrm{d}_{\mathrm{s}}=280 \mu \mathrm{m}$. There, the density of hot gases is typically $0.31 \mathrm{~kg} / \mathrm{m}^{3}$ near ambient pressure and it is $2.5 \mathrm{~kg} / \mathrm{m}^{3}$ at $8.1 \mathrm{~atm}$. All plastic grit and glass powders used have identical particle-size-distribution (PSD) relative to the mean Sauter diameter.

The circulating fluidized bed of $7 \mathrm{~m}$ height and $20 \mathrm{~cm}$ riser diameter is described in (1). Its unique ability is to recycle any mixture of inert fluidization gases, thus setting all five dimensionless numbers at the desired values. Static pressure is measured using 35 taps mounted flush along the height of the riser. Experimental conditions are listed in (3).

\section{VERTICAL GAS PRESSURE PROFILES}

In the relatively dense suspensions of circulating fluidized beds, vertical profiles of static gas pressure reveal the underlying character of the flow. Figure 1 illustrates the effects of Froude number on vertical profiles of static gas pressure. There, the elevation is shown as a fraction of riser height, $\mathrm{z}^{\dagger} \equiv \mathrm{z} / \mathrm{H}$, and the static gas pressure relative to the riser top is made dimensionless as $\mathrm{p}^{\dagger}=\left(\mathrm{p}-\mathrm{p}_{\text {top }}\right) / \rho_{\mathrm{s}} \mathrm{gD}$.

Surprisingly, the Froude number hardly affects the pressure gradient in the upper riser. However, as expected, smaller Froude numbers produce higher acceleration regions, particularly at high loadings. The data in (3) under "atmospheric" conditions also betray minimal effects of the Froude number on the pressure gradient . However, as our later analysis will explain, this insensitivity to the Froude number is not universal, although the corresponding dependence is generally weak. Thus, as Fig. 1 shows, the solid loading is the operational parameter with greatest effect on the pressure gradient in the upper riser.

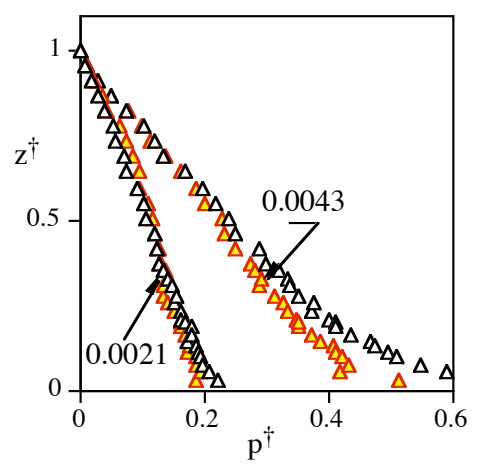

Figure 1 Effects of Froude and loading in "pressurized" tests. The open and solid triangles represent $\mathrm{Fr}=102$ and 132, respectively. Conditions are $\mathrm{L}=1315$, $\mathrm{Ar}=370, \mathrm{R}=600$ and $\mathrm{M} / \mathrm{R}$ as shown.
In the upper riser, the pressure gradient, and thus the solid volume fraction, are generally smaller for denser gases. This confirms the trends observed by Karri and Knowlton (ㅁ). At the base, because in the inertial regime denser gases produce larger drag, the height of the acceleration region is considerably smaller for "pressurized" tests.

Finally, to study the effects of Ar alone, we simulated pressurized risers of nearly identical scale but widely unequal Archimedes numbers. We found that this difference did not affect vertical pressure profiles in the upper riser over a wide range of conditions.

\section{ANALYSIS}

Our observations of the relative insensitivity of the pressure gradient to Ar, Fr and L in the upper riser suggest a simple interpretation. From the time-average momentum balances in ( $\underline{4})$, we write fully-developed, axisymmetric and steady balances of momentum in the upper riser. For the gas, 
$-\varepsilon \frac{d p}{d z}=\rho \varepsilon g+F-\frac{1}{r} \frac{d(r \tau)}{d r}$

and for the solids,

- $(1-\varepsilon) \frac{\mathrm{dp}}{\mathrm{dz}}=\rho_{\mathrm{S}}(1-\varepsilon) \mathrm{g}-\mathrm{F}-\frac{1}{\mathrm{r}} \frac{\mathrm{d}(\mathrm{rS})}{\mathrm{dr}}$,

where $\mathrm{z}$ and $\mathrm{r}$ are the upward vertical and radial coordinates, respectively, and $\tau$ and $\mathrm{S}$ are the gas and solid stresses on surfaces at constant radius. Upon adding Eqs. [6] and [7], and averaging across the pipe, we find

$-(\mathrm{dp} / \mathrm{dz})=\left[\rho_{\mathrm{S}}(1-\bar{\varepsilon})+\rho \bar{\varepsilon}\right] \mathrm{g}-(4 / \mathrm{D})\left(\mathrm{S}_{\mathrm{W}}+\tau_{\mathrm{w}}\right) \approx\left[\rho_{\mathrm{S}}(1-\bar{\varepsilon})+\rho \bar{\varepsilon}\right] \mathrm{g}$,

where $S_{\mathrm{W}}$ and $\tau_{\mathrm{W}}$ are shear stresses exerted by the wall on the solid and gas phases, respectively. Because these stress terms decrease with $\mathrm{D}$, their contributions to the pressure drop become negligible in relatively large risers.

Upon averaging Eq. [6] and eliminating dp/dz with Eq. [8], we find

$\bar{\varepsilon}(1-\bar{\varepsilon}) g\left(\rho_{s}-\rho\right)=\bar{F}$.

It remains to describe the volumetric drag. At any radial position, it is proportional to the solid volume fraction and the local relative velocity between the two phases,

$\mathrm{F}=\left(\rho_{\mathrm{s}} / \mathrm{T}\right)(1-\varepsilon)(u-v)$,

where $u$ and $v$ are the interstitial gas and particle velocities, respectively, and $\mathrm{T}$ is the particle relaxation time defined in terms of the drag force on one particle by

$\left(\rho_{\mathrm{s}} / \mathrm{T}\right)=\mathrm{C}_{\mathrm{d}} \mathrm{u}_{\text {slip }}(3 \rho / 4 \mathrm{~d})$,

where $\mathrm{C}_{\mathrm{d}}$ and $\mathrm{u}_{\text {slip }}$ are, respectively, the drag coefficient and mean slip velocity experienced by a particle in the presence of its neighbors and $\mathrm{d}$ is its diameter.

We introduce several simplifications to evaluate the average drag force. Following $(\underline{3})$, we equate the particle diameter $d$ to the product of the particle sphericity and mean Sauter diameter, $\mathrm{d}=\phi \mathrm{d}_{\mathrm{s}}$. Then, as Littman, et al (6) proposed, a balance of weight and drag similar to that involved in calculating the terminal velocity suggests a relation between the drag coefficient and the slip velocity,

$(1 / 2) \rho \stackrel{2}{2} \mathrm{u}_{\text {slip }} \mathrm{C}_{\mathrm{d}}=(2 / 3) \rho_{\mathrm{s}} \mathrm{g} \mathrm{d}[1-(1 / \mathrm{R})]$.

Crucially, we further assume that $\mathrm{u}_{\mathrm{slip}}$ scales with the superficial gas velocity through a slip coefficient $\lambda$ to be determined,

$\mathrm{u}_{\mathrm{slip}}=\lambda \mathrm{u}$

and that both $\lambda$ and $\mathrm{T}$ are uniform across the riser.

Eliminating the velocities with definitions of $\mathrm{u} \equiv \overline{u \varepsilon}$ and $\mathrm{G} \equiv \rho_{\mathrm{s}} \overline{v(1-\varepsilon)}$, the average volumetric drag becomes

$\overline{\mathrm{F}}=\left(\rho_{\mathrm{s}} / \mathrm{T}\right) \mathrm{u}[(\alpha / \bar{\varepsilon})-(1+\mathrm{M} / \mathrm{R})]$, 
where $\alpha \equiv \bar{\varepsilon} \quad \bar{u} \quad / \overline{u \varepsilon}$ captures the spatial correlation between the radial profiles of interstitial gas velocity and voidage. Finally, combining Eqs. [11] through [13] and substituting the resulting expression for T in Eqs. [9] and [14], the force balance becomes

$1-\bar{\varepsilon}(1+\mathrm{M} / \mathrm{R})=\lambda \bar{\varepsilon}^{2}(1-\bar{\varepsilon})-(\alpha-1)$.

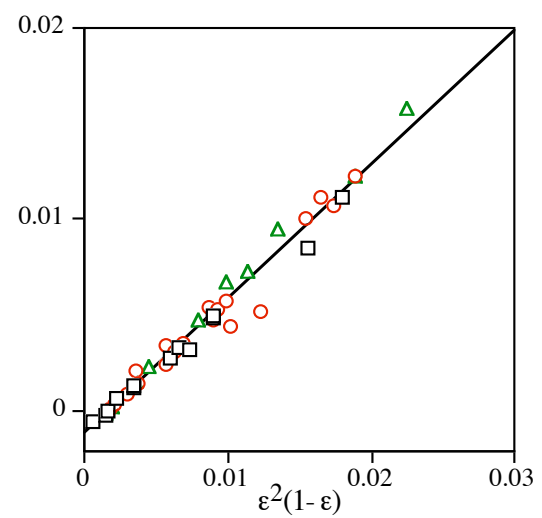

Figure 2 Plot of $1-\bar{\varepsilon}(1+\mathrm{M} / \mathrm{R})$ vs $\bar{\varepsilon}^{2}(1-\bar{\varepsilon})$ for the "pressurized" tests with $\mathrm{R}=600$. The squares, triangles and circles are $(\mathrm{L}=2029, \mathrm{Ar}=356),(\mathrm{L}=1315, \mathrm{Ar}=370)$, and $(\mathrm{L}=2092, \mathrm{Ar}=95)$. The best fit is $\lambda=0.70 \pm 0.02 ; \alpha-1=0.0011 \pm 0.0002$.
We invoke data from a wide range of conditions to gauge the merit of the approach leading to Eq. [15]. In addition to our own results and those in (ㄱ) $)$ we borrow from Wang, Rhodes and Gibbs (7), who reported solid volume fractions in the upper riser of an atmospheric CFB operated at temperatures of 20,270 and $550^{\circ} \mathrm{C}$.

As Fig. 2 shows, a plot of $1-\bar{\varepsilon}(1+\mathrm{M} / \mathrm{R})$ against $\bar{\varepsilon}^{2}(1-\bar{\varepsilon})$ is a single straight line of slope and intercept independent of M/R. Therefore, Eq. [15] captures the dependence of the crosssectional average solid volume fraction on $\mathrm{M} / \mathrm{R}$ in a simple way, despite considerable variations of that quantity.

\section{FLOW REGIMES OF THE UPPER RISER}

The slip coefficient $\lambda$ varies widely among the tests considered here. We now examine its behavior in two limiting flow regimes. From Eqs.[1], [12] and [13], we obtain $\lambda^{2}=(4 / 3)(\mathrm{R}-1) /\left[\mathrm{C}_{\mathrm{d}} \mathrm{Fr}^{2}\right]$.

Therefore, in this simple treatment, $\lambda$ is largely determined by the behavior of $C_{d}$.

As Fig. 2 shows, $\lambda$ is remarkably independent of the average solid loading. If the flow consisted of a homogeneous suspension, then the drag force would vary with the average distance between neighboring particles (8). In that case, the loading would affect $\lambda$ appreciably. Instead, particles congregate in clusters that become more numerous as loading grows without changing their basic momentum interaction with the surrounding flow.

Because the ubiquitous particle clusters involve a majority of solids and because their concentration is much higher than the surrounding suspension, they dominate the average of any quantity proportional to the solid volume fraction. As a result, the drag that they experience likely governs the mean momentum exchange between the two phases and, consequently, the average gas pressure gradient.

Within an individual cluster, the largest slip likely occurs at the periphery. Because particles in the cluster's interior entrain the surrounding gas at nearly the same velocity, they possess a much smaller slip. In this description, the only appreciable drag is experienced by particles distributed over a region a few particle diameters thick at the cluster's periphery. There, an estimate of the mean strain rate is $\gamma \sim \mathrm{u}_{\text {slip }} / \mathrm{d}$. The ability of clusters 
to follow changes in the surrounding gas velocity fluctuations diminishes with increasing values of a Stokes number that is the product of the mean strain rate and the particle relaxation time,

$\mathrm{St}=\gamma \mathrm{T}=(4 / 3) \mathrm{R} / \mathrm{C}_{\mathrm{d}}$.

Combining Eqs. [16] and [17], the slip coefficient scales as

$\lambda \approx \sqrt{\mathrm{St}} / \mathrm{Fr}$.

We expect two asymptotic limits in the drag. In the first, viscous forces dominate. By analogy with a single particle, we assume that the resulting drag coefficient is

$\mathrm{C}_{\mathrm{d}}^{0} \sim 24 / \operatorname{Re}_{\text {slip }}$,

where the superscript denotes the viscous limit and

$\operatorname{Re}_{\text {slip }} \equiv \mathrm{u}_{\text {slip }} \rho \mathrm{d} / \mu=\lambda \operatorname{Fr} \sqrt{\operatorname{Ar} / \mathrm{R}}$.

In the limit of vanishing $\mathrm{Re}_{\text {slip }}$, we estimate $\mathrm{St}$ by combining Eqs. [17] and [19],

$\mathrm{St}^{0} \approx \mathrm{R} \mathrm{Ar} / 18^{2}$

and, at higher $\operatorname{Re}_{\text {slip }}$, we write

$\mathrm{St} \sim \mathrm{St}_{\mathrm{d}}^{0} /\left(1+0.15 \mathrm{Re}_{\text {slip }}^{0.7}\right)$

where the expression in parentheses captures effects of gas inertia for an isolated particle with $\operatorname{Re}_{\text {slip }}<800(\underline{9})$. Because the momentum interaction between the phases involves groups of particles, we do not expect that the numerical factors in Eqs. [19] and [21] are accurate. However, the resulting trends are instructive.

Gas inertia dominates the other asymptotic limit. If for simplicity we assume that the corresponding drag coefficient is constant,

$\mathrm{C}_{\mathrm{d}} \sim \mathrm{C}_{\mathrm{d}}^{\infty}$

then, from Eq. [16], the slip coefficient depends mainly upon the density ratio,

$\lambda \approx(1 / \mathrm{Fr}) \sqrt{\left(4 / 3 \mathrm{C}_{\mathrm{d}}^{\infty}\right) \mathrm{R}}$.

In this expression, note that $\lambda$ is now independent of the Archimedes number.

Wang, et al (7) argued that their flows were chiefly affected by the gas viscosity. Similarly, because our atmospheric tests also exhibit small values of $\mathrm{Re}_{\text {slip }}$, we expect that they conform to the viscous limit. As Fig. 3 shows, the corresponding dependence of $\lambda$ upon $\sqrt{\mathrm{St}}$ is qualitatively captured by Eq. [18]. However, that Eq. clearly exaggerates the role of the Froude number. In fact, at the high Stokes of our atmospheric tests, $\lambda$ is insensitive to Fr. In the experiments of Wang, et al (7) at $550^{\circ} \mathrm{C}$, the Stokes number is lower, while $\lambda$ displays a relatively weak dependence of order $\mathrm{Fr}^{-0.5}$. 


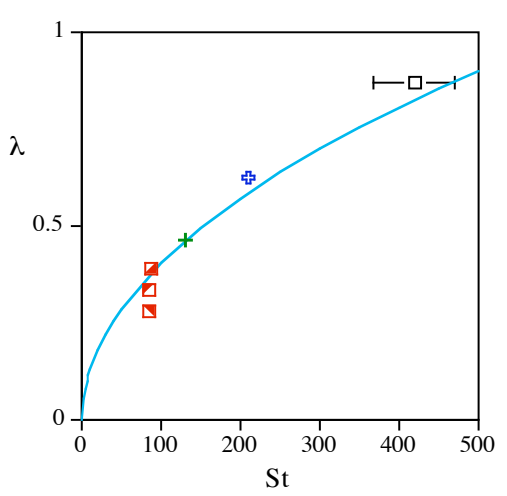

Figure 3 Variations of $\lambda$ in the viscous limit. The square and bar show the range of superficial velocities in our atmospheric tests. Other data is from (7). The line is a best fit following Eq. [18].
These observations suggest that high Stokes numbers limit the effects of the superficial gas velocity upon the mean cluster drag in a similar way that they inhibit the response of a single particle to gas velocity fluctuations.

In contrast, because they exhibit greater values of $\mathrm{Re}_{\text {slip }}$, the pressurized experiments are likely dominated by gas inertia. In fact, our measurements of $\lambda$ under "pressurized" conditions are quite insensitive to the Archimedes number, as Eq. [24] predicts.

\section{ACKNOWLEDGMENTS}

This work was funded by the University Coal Research Program of the US Department of Energy, Pittsburgh Energy Technology Center under grants DE-FG2293PC93216 and DE-FG22-95PC95228, and by the International Fine Particle Research

Institute. The authors are grateful to Professors Leon Glicksman and Sankaran Sundaresan for stimulating discussions.

\section{$\underline{\text { REFERENCES }}$}

1. Louge M., Bricout V. and Martin-Letellier S., Chem . Eng. Sci. (1998), in press.

2. Glicksman, L.R., Hyre, M. and Farrell, P., Int. J. of Multiphase Flow 20, 331 (1994).

3. Chang, H. and Louge, M.Y., Powder Tech. 70, 259 (1992).

4. Anderson, T.B. and Jackson, R., Ind. Eng. Chem. Fundamentals 6, 527 (1967).

5. Karri, S. B. R, and Knowlton, T. M., Proceedings of Circulating Fluidized Beds V, Paper DB15, Beijing, China, May 28 - June 1 (1996).

6. Littman, H., Morgan, M.H., Paccione, J.D., Jovanovic, S.D. and Grbavcic, Z.B. Powder Tech. 77, 276 (1993).

7. Wang, X.S., Rhodes, M.J., and Gibbs, B.M., Chem. Eng. Sci. 50, 2441 (1995).

8. Foscolo, P. U. and Gibilaro, L. G. Chem. Eng. Sci. 39, 1667 (1984).

9. Clift, R., Grace, J.R. and Weber, M.E., Bubbles, drops and particles, Academic Press (1978). 